\title{
El pensamiento complejo y los desafíos de la educación del siglo XXI
}

Wilmar Aníbal Peña Collazos ${ }^{1}$

\section{Resumen}

La educación en América Latina reclama hoy un nuevo paradigma acorde con nuestra mentalidad, desde un espectro pluridimensional; exige una orientación al reconocimiento de su sabiduría ancestral, a la integración de sus creencias, sus sensibilidades, su ciencia y su sentido vital, para generar una comprensión interna desde el uso apropiado de todas sus facultades, que propicie la auto-ecoorganización y la sabiduría de nuestros ancianos y antepasados. Edgar Morin pretende que, a partir de la "reforma de la educación" se puedan articular los saberes para lograr el pensamiento complejo nece sario para que las nuevas generaciones respondan a las exigencias del nuevo milenio. Esto saberes son: el científico, el lógico, el simbólico, el poético y el demencial complejizador. Éstos harán parte de la formación integral indispensable para generar el entendimiento interdisciplinario y holístico que nos exige hoy el mundo globalizado.

Filósofo de la Pontificia Universidad Javeriana y Magíster en Filosofía Latinoamericana de la Universidad Santo Tomás. Candidato a Doctor en Filosofía por la Universidad Javeriana. Docente de la Universidad Externado de Colombia y Miembro de la Asociación Colombiana de Pensamiento Complejo. Correo de contacto: pc.wilmar@gmail.com 
Es necesaria una reforma del pensamiento desde la reforma de la educación, lo cual, a su vez, incide en la transformación del pensamiento. El pensamiento complejo reclama una educación que valore la afectividad y asuma el error como medio propicio para el cambio. Desde la acertividad positivista, la lógica occidental preconizó un saber dogmático y controlador, que negó el carácter lógico de la pasión, los deseos, las emociones, los sentimientos y la vida afectiva. De acuerdo con el pensamiento complejo, la educación debe dedicarse a identificar las cegueras del entendimiento, en vigilante autocrítica que admita los propios errores, que procure la incertidumbre racional y que reconozca la memoria cultural de nuestras naciones.

Este artículo es un resultado asociado al grupo de investigación Liderazgo, de la Universidad Militar Nueva Granada, es cual está reconocido por Colciencias y clasificado en Categoría B.

\section{Palabras clave}

Complejidad, educación compleja, auto-eco-organización, saberes múltiples, ecología, cibernética, sistémica.

\section{Abstract}

Today, education in Latin America calls for a new paradigm in accordance to our way of thinking from a multidimensional spectrum. It calls for an education that is geared towards the recognition of Latin America's millenary wisdom, the integration of its beliefs, sensibilities, science and life thrust, in order to generate a self understanding that stems from the appropriate use of all faculties that promotes auto-eco-organization and the wisdom of our elders. With Edgar Morin, we believe that a number of forms of knowledge can be articulated in order to achieve the complex thought necessary for new generations to answer the challenge of the new millennium, stemming from a reform of education. These forms of knowledge are: scientific, logical, symbolic, poetic and complex. They would be part of the integral education necessary for interdisciplinary and holistic understanding that our current globalized world demands.

It is necessary to reform thought by reforming education, which, through a feedback loop, reforms thought. Complex thought claims an education that values affectivity and assumes errors are good means towards change. Western logic, through positivist assertivity, adopted a dogmatic and controlling knowledge that denied the logical character of passions, desires, feelings and emotions. Education in complex thought must strive to identify blind spots in thought, must be vigilant in a self criticism that admits its own mistakes, that seeks rational incertitude and recognizes the memory of our nations.

\section{Key words}

Complexity, complex education, auto-eco-organization, multiple forms of knowledge, ecology, cybernetics, systems. 


\section{Introducción}

Al abordar nuestro conocimiento sobre el paradigma educativo del pensamiento complejo, nos planteamos la siguiente pregunta: ¿cómo abrirnos a este nuevo paradigma del conocimiento a partir de las estructuras que ya tenemos, con el fin de proponer una educación auténtica desde nuestras estructuras mentales y culturales? (Gutiérrez, 2001). Hoy, la educación se constituye en un instrumento poderoso para realizar el cambio cultural. Con ella se buscan nuevos horizontes, desde una concepción integradora del entorno económico, cultural, social y político. Es un proceso que tiene sentido, por cuanto brinda el paso de la heteronomía a la autonomía; por eso los fundamentos de este nuevo paradigma se zanjan en el aprendizaje autónomo y significativo.

El espíritu humanista e ilustrado del Siglo de las Luces promovió la confianza en el individuo y la razón. No será posible dar un paso significativo a los ideales de la posmodernidad sin antes recuperar las pretensiones de Kant, al darle al sujeto la supremacía, por lo cual el hombre moderno deberá alcanzar la mayoría de edad y pensar por sí mismo, ser autónomo y con principios humanos muy sólidos. En realidad, la clave de este nuevo proceso es el conocimiento; no un conocimiento solipsista y aislado, sino integrador y compartido, comunicado, representado, lo cual está necesariamente ligado a la educación, que es, a la vez, la clave de la innovación, producto de la investigación.

Las distintas culturas adquieren diferentes lógicas y modelos de pensamiento . Los indígenas tuvieron una lógica distinta a la lógica que impusieron los españoles en la conquista de Abyayala (Páramo, 1989). América Latina ha adquirido un constructo lógico especial, producto de la hibridación cultural; es decir, poseemos un calor cultural distinto: la dinámica de una construcción lógica en tensión y perplejidad, propicia para la complejidad.

La manera de usar los sentidos y el pensamiento se interrelacionan y se explican en la transición de la oralidad a la escritura. Mientras la oralidad se fo- calizaba en el oído y la boca, la escritura enfatiza el ojo y la mano. Debido a este cambio, la civilización occidental ha utilizado el lenguaje escrito como fuente de desarrollo del conocimiento y del propio pensamiento. La racionalidad es explicitada a través del lenguaje que organiza y fija el conocimiento. Por este motivo, McLuhan considera que toda la civilización occidental ha estado centrada en el desarrollo del hemisferio izquierdo del cerebro², y ello ha contribuido al desarrollo del razonamiento cuantitativo. Por el contrario, la cultura oriental ha mantenido el espacio acústico proyectado hacia el hemisferio derecho, desarrollando un pensamiento más cualitativo y holista. Asimismo, el hombre latinoamericano está avocado al reconocimiento de su sabiduría ancestral, a la integración de sus creencias, sus sensibilidades, su ciencia y su sentido, para generar una comprensión interna desde el uso apropiado de todas sus facultades. El hombre latinoamericano reconstruye su saber y para este cometido es propicio el carácter educativo del pensamiento complejo.

Edgar Morin advierte que sólo a partir de una "reforma de la educación" se podrán articular los saberes para lograr el pensamiento complejo necesario para que las nuevas generaciones respondan a las exigencias del nuevo milenio. Estos saberes son: el saber científico, el lógico, el simbólico, el poético y el demencial complejizador. Éstos harán parte de la formación integral indispensable para el entendimiento interdisciplinario y holístico que hoy nos exige el mundo globalizado. Para Morin, es claro que se requiere la reforma del pensamiento para reformar la educación, lo cual incide a su vez en la reforma del pensamiento (Morin, 1999).

El pensamiento complejo reclama una educación que valore la afectividad y asuma el error como un medio propicio para el cambio. La lógica occidental, desde los aciertos positivistas, preconizó un saber dogmático, controlado desde la certidumbre,

2 En el hemisferio izquierdo predominan los aspectos racionales (pensamiento lógico-matemático, secuencial, analítico, etc.). En cambio, en el derecho predominan los aspectos emocionales, intuitivos, la visión holística. 
dividido y cuantitativo; se evadieron los deseos, las emociones, los sentimientos, las pasiones, la vida afectiva como ejes del error. La educación, desde el pensamiento complejo, debe dedicarse a identificar las cegueras del entendimiento en vigilante autocrítica, que admita los propios errores, que procure la incertidumbre racional y que reconozca la memoria cultural de nuestras naciones. La educación ha de tener en cuenta que el juego de teorías se construye no desde la verdad, sino desde la dialéctica del error y la verdad; los paradigmas se imponen desde el inconsciente colectivo que admite los cambios en la ciencia y el proceso en la investigación. La educación debe encaminarse para afrontar la incertidumbre y asumir los riesgos para atreverse a comprender a la persona humana.

El conocimiento, que se nutre de memoria biológica y cultural, imprime una marca, un estigma o "imprinting" de las primeras experiencias cognitivas que dan carácter e identidad, y estimula la toma de conciencia de realidades antropológicas y sociológicas profundas hic et nunc (aquí y ahora). Para Morin, es necesario entender lo biológico como elemento ordenador que interactúa con el pensamiento cultural. Desde este punto de partida, propone un cambio sustancial de la manera como se ve, se ordena y se llega al conocimiento. Para lograrlo, es necesario cambiar o identificar de manera diferente lo que llamamos conocimiento, por ello plantea la reforma del conocimiento del conocimiento. Será necesario integrar al sujeto en su conocimiento con un bucle de incertidumbre del conocimiento, es decir, un conocimiento que procure las condiciones noológicas ${ }^{3}$ de teorías abiertas, condiciones socioculturales de las ideas que permean la cultura y condiciones bioantropológicas que integren las operaciones cerebrales mentales a los problemas fundamentales del mundo, del hombre y la sociedad. Se propone,

3 Desde el paradigma de la complejidad, la noosfera es la esfera del nous o del espíritu. A partir del nous, cada sociedad construye sus creencias e ideas mentales, lo cual configura los sistemas de creencias, los rituales y las acciones prácticas que se despliegan en la cotidianidad de una cultura. entonces, un metasistema en el que se incluyan las ramas del saber y su eje necesariamente recae en la educación. Este nuevo conocimiento sería "auto-trans-metaorganizador", pues será un conocimiento que se autodetermine, se explique en sí mismo como bucle del pensamiento complejo y, por tanto, sería meta-ordenador.

Los elementos articuladores de una cultura son la educación, la tradición, el lenguaje. Ahora bien, la dinámica de la cultura no sólo depende de los tres elementos anteriores, porque también representa una tensión entre tradición e innovación. Además, todo conocimiento depende del contexto cultural, social e histórico. De hecho, la psicología cognitiva plantea que un conocimiento no es pertinente si no muestra que es capaz de interpretar y transformar el contexto. Por esta razón, en la actualidad resulta infructuoso hablar de problemas vitales sin contextualizarlos en las disciplinas, de modo que contribuyan a la comprensión de las situaciones culturales y sociales.

Los grandes problemas que marcan el futuro de la humanidad son transversales, trasnacionales, multidimensionales, transdisciplinarios. La reforma de la enseñanza es crucial para la reforma del pensamiento, lo cual constituye una tarea histórica y holográmica en la que no sólo la parte está en el todo; sino que el todo, en cierto modo, está en la parte. Asimismo, la transversalidad de los problemas exige transversalidad investigativa compleja. Resulta infructuoso hablar de problemas vitales sin contextualizarlos en sus disciplinas.

Entonces, educar se convierte en una tarea compleja de cultivar la integridad humana, mediante prácticas formativas que promuevan, desde la interdisciplinariedad, la lectura creativa de los contextos culturales y sociales. No se trata de suprimir las disciplinas para hablar de todo y de nada a la vez, falseando la realidad; sino de relacionar los aportes de todos los saberes posibles para la solución de problemas fundamentales, complejos, hic et nunc (aquí y ahora). La educación debería propiciar una "inteligencia general", multidemensional al contexto, con una concepción global. 
Nuestra educación tradicional nos ha enseñado a dividir, separar y ver sólo lo que parcialmente corresponde a problemas encerrados en sí mismos, sin captar la totalidad. Somos, pues, esclavos de una falsa racionalidad que nos impide comprender la multidimensionalidad. Es así como en el mundo se han producido enormes progresos científicos y tecnológicos, pero se han olvidado los problemas globales y complejos.

No se trata de abandonar el conocimiento de las partes por el conocimiento del todo, sino más bien de integrar y relacionar los distintos factores de la condición humana. Interrogar a ésta es indagar por nuestra situación en el mundo. La educación debería mostrar el destino vital, sublime y ecológico del hombre, pues en el seno de singularidad que nos es propia llevamos toda la humanidad, la civilización, el cosmos total. Una vocación esencial de la educación es el estudio de la complejidad humana; ella conduce a la toma de conciencia de la condición común a todos y la necesaria diversidad de las culturas. Para Morin, es imposible comprender la realidad si no se intenta comprender todas las instancias posibles de lo real en distintos niveles y desde distintos puntos de vista, pues la realidad debe concebirse desde distintos saberes, interdisciplinariamente, como sistemas cuyos elementos son interdependientes.

El error fundamental de la ciencia tradicional consistió en poner el mundo como objeto de conocimiento fuera del sujeto. Un conocimiento que analiza, divide, describe y explica procesos sin poder comprender holísticamente la realidad. En la investigación social el problema no está por fuera del acto del conocimiento; se integra al proceso creativo, es decir, sólo podemos comprender aquello de lo cual formamos parte.

La identidad de la persona es compleja porque es unitas y a la vez multiplex, el yo es uno y a la vez corresponde al otro. Cada quien, a través de su cuerpo, gesticula, simboliza y expresa un lenguaje que se pronuncia desde la realidad subjetiva que trasciende sus propias barreras de intimidad. Cada sujeto es un pueblo que se expresa como individuo y como otredad cultural. Además, el conocimiento se articula dinámicamente en el "calor cultural", bajo condiciones de inestabilidad o crisis que exige cambios profundos. Es necesaria la tensión de espíritus complejos, reflexivos, que generen ideas nuevas, con autonomía y crítica interiorizada.

El individuo se manifiesta en la cultura y toda cultura es producto de la comunicación, que requiere la creación de instrumentos y signos que le permitan manifestarse al sujeto. No se puede tener certeza que una persona ha sellado el proceso de formación de un concepto, aun cuando sea capaz de verbalizarlo. Es necesario, cambiar las maneras de pensar, construir y deconstruir; ir desde modelos simples a modelos complejos, inmersos en la experiencia sociocultural que se transforma permanentemente como articulación de saberes y contextos plurales.

La investigación didáctica ha privilegiado factores como ideas previas, metacognición y transposición, necesarios para que los estudiantes puedan aprender. En la vivencia de aprender creando se asocia un sentido nuevo, una mayor fluidez del espíritu y del cuerpo, la producción simbólica y la verbal, asociadas a la habilidad para enriquecer categorías conceptuales apropiadas y reconstruidas por los mismos estudiantes en su proceso de interiorización de la ciencia. Esto es positivo porque nos otorga matices para interpretar la realidad desde la visión particular y desde el repertorio cognitivo singular; allí se convoca la multidisciplinariedad que propone nuevas luces y, a la vez, dispone nuevos acertijos.

Para pensar una propuesta pedagógica compleja será necesario plantear una gestión del conocimiento con nuevas tecnologías, sin caer en el peligro de ahogar los fines del saber, la creatividad, la conceptualización y la interacción profesor/alumno desde la propuesta instrumental de los medios, lo cual sería fatal, algo así como instrumentalizar el conocimiento, los afectos, la sensibilidad y la creatividad. Hacia el futuro se consideran grandes e ilusorias profecías de tipo milenarista: una nueva ortodoxia parece imponerse: la creencia en que la 
Internet, la Intranet, la multimedia o los mass media va a solucionar todos los problemas didácticos y educativos. Junto con la progresión cognitiva y la creación de entornos de aprendizaje abiertos y a distancia, afloran instrumentos metacognitivos que permitirán al alumno sostener un intercambio distinto: el estudiante interactuará de acuerdo con los determinismos del lenguaje, los símbolos y los imaginarios que se proyecten a través de los medios, de una pantalla, de un video, conferencias informatizadas, etc. Si bien es cierto que la educación multimedial propone un distanciamiento individualista y menos corporal, la sociedad día a día se perfila hacia la atomización de la información que se encapsula como paquetes informáticos que podrán viajar de un lugar a otro del planeta en cuestión de segundos. Sin embargo, la tarea de la educación es compleja frente a las múltiples redes de información: es necesaria una tarea crítica de interpretación, apropiación coherente y pertinente de la información para construir un discurso útil; un conocimiento que responda a nuestras necesidades más profundas.

Las instituciones educativas y las maneras de educar han ido cambiando. Uno de los principales retos que tiene la universidad en nuestros días se refiere a una educación más apropiada al conocimiento y a la nueva manera de concebirlo como fruto de las transformaciones de la sociedad y la cultura. En efecto, éste se concibe como fuente de poder, como la condición para el buen funcionamiento tanto de las estructuras sociales e individuales; todo gira alrededor de él, y por eso esta sociedad del conocimiento es entendida como "la capacidad de producir, almacenar, transmitir y recuperar la información generada por los soportes de la información y de la comunicación" (Ramón, 1999: 50).

\section{La sociedad del conocimiento y las nuevas tecnologías}

La sociedad del conocimiento se entiende desde la información y el aprendizaje a partir de ella. En la actualidad, el mundo se halla en la dinámica de aprender de manera progresiva. De acuerdo con Pozo,

\begin{abstract}
El aprendizaje continúa más allá de los ámbitos educativos, no sólo a lo largo de nuestra vida, debido a la demanda de un aprendizaje continuo en el ejercicio profesional, sino también a lo ancho de nuestros días, ya que las actividades formativas alcanzan, podríamos decir, que en paralelo a las necesidades educativas y de formación profesional, a casi todos los ámbitos de la vida social [...]. Nuestra interacción cotidiana con la tecnología nos obliga a adquirir continuamente nuevos conocimientos y habilidades (1999: 38).
\end{abstract}

La educación participó de las contradicciones de fin de siglo y participa de las del inicio de este nuevo milenio. Por una parte, somos espectadores de los cambios trascendentes y acelerados que supone, entre otros aspectos, el desarrollo de la tecnología digital, que está transformando muchos aspectos de la vida diaria. Las comunicaciones a escala mundial amplían nuestros horizontes culturales y cambian las pautas de competencia de las economías. Como dice un reporte reciente, "la vida moderna ofrece nuevas oportunidades y opciones a los ciudadanos, pero también entraña mayores riesgos e incertidumbres. La gente tiene la libertad de adoptar diversos estilos de vida, pero también la responsabilidad de dar forma a sus propias vidas" (Comisión Europea, 2000).

Sin embargo, las nuevas posibilidades que ofrece la educación no están disponibles para todos; por el contrario, los procesos de mundialización o globalización económica parecen ahondar las diferencias. El Informe Delors, de la Unesco, señaló que la educación es la "vía al servicio del desarrollo humano más armonioso". Pero, ¿cómo vivir racionalmente en esta aldea planetaria si no aprendemos a convivir en las aldeas locales? La educación se encuentra en la perspectiva del nacimiento de una sociedad del conocimiento, y en este contexto, la educación tiene la misión 
de "permitir a todos sin excepción fructificar todos sus talentos y todas sus capacidades de creación, lo que implica que cada uno pueda responsabilizarse de sí mismo y realizar su proyecto personal" (Delors, 1997: 12).

En otros tiempos -por ejemplo, durante el Antiguo Régimen- la información era restringida y el saber se consignaba en latín para el manejo y conocimiento de pocas personas privilegiadas. El aprendizaje correspondía a un modelo administrativo centralizado y burocrático; el saber pertenecía a unos pocos que podían dirigir los procesos, prescribían qué enseñar, en dónde, cuándo y a quiénes les estaba permitido aprender formalmente. En la actualidad, tal dinámica ha cambiado: el conocimiento ha perdido el carácter centralizado que había tenido por tradición, lo cual ha propiciado el despertar reflexivo y crítico de comunidades académicas que han dado paso a la diversificación de teorías y modelos educativos. Gracias a la tecnología, ahora la información tiende a distribuirse más democráticamente; se abren las puertas de la información mediante múltiples canales y medios de comunicación, y se encuentra de todo: en algunos casos con precisión, ética, rigor crítico y sentido humano, en otros, con superficialidad y engaño. Se trata pues de poner filtros, procesar y sistematizar la información para aprehender.

La tecnología es el resultado de la creatividad y del aprendizaje social e innovativo de personas asociadas, de grupos y sociedades en donde ella se produce. Es el resultado de un proceso social e histórico que a la vez la determina, y el producto de estructuras mentales dadas que no se pueden transferir, de valores culturales determinados que no se pueden comprar y de formas peculiares de vivir, pensar, sentir y obrar, difíciles de imitar o reproducir (Ramón, 1999: 50).

Las nuevas tecnologías se abastecen de la información y el conocimiento, Lo más importante para poder ser fuertes es el conocimiento. De esta ma- nera, la educación y el conocimiento se convierten en los abastecedores de la tecnología de la información. De acuerdo con Latorre y Suárez (2000), la tecnología "invade toda la civilización imponiéndole tradiciones y valores, y sentido de las creencias, intereses y tradiciones de una región debido a la invasión no sólo de los bienes de consumo, sino de la industria de la cultura".

Las claves innovadoras de la educación estriban en formarse apropiadamente para la adaptación a nuevas situaciones y tecnologías que van cambiando nuestros hábitos y costumbres. El conocimiento es dinámico, continuamente se renueva, y quien no actualice sus conocimientos con verdadero espíritu autónomo y significativo, es decir, si alguien no sabe dónde ni cómo buscar la información, habrá fracasado en esta nueva era del manejo apropiado de la información y el conocimiento.

El maestro adquiere paulatinamente la tendencia a buscar sus mayores soportes en las Tecnologías de Información y Comunicación (TIC). La cuestión de una crítica de la comunicación pasa por una crítica de las nuevas tecnologías de la información y la educación ha de considerar de qué manera llega el conocimiento y la apropiación del saber. La labor educativa no es exclusividad de los maestros de profesión; la ciudad y la sociedad en general reclaman el derecho de convertirse en educadores y gestores del cambio cultural.

\section{Las condiciones están dadas}

La pregunta medular de la comunidad educativa es: ¿qué es lo que necesitamos aprender?, es decir, ¿cuáles son los aprendizajes necesarios para enfrentar las necesidades y demandas que nos plantea el mundo contemporáneo? La pregunta es pertinente, porque lo que hacemos (o no hacemos) hoy en educación tendrá algún impacto en lo que será nuestro país y el mundo en el futuro, ya que las generaciones que se forman hoy seguirán viviendo y desplegando su acción en las siguientes décadas. 
A la luz de los desarrollos actuales, es cada vez más evidente que la educación es una tarea compleja que supone la interacción de diversos factores. En la formación de las personas intervienen múltiples elementos enmarcados en diferentes relaciones sistémicas: sociedad, familia, agentes biológicos y culturales. El Informe Delors desarrolló, además, un concepto clave: la educación es un proceso que dura toda la vida, lo cual supone que no está reducido a la formación de las generaciones jóvenes, sino que es un proceso continuo que debe atravesar toda la vida de una persona. A esto ha contribuido también el hecho de que los conocimientos se transforman cada vez más rápido, de manera tal que lo que uno aprende en un momento de su vida debe ser revisado poco tiempo después, dada la amplia movilidad y el enriquecimiento continuo de las disciplinas. Esto ha supuesto la ampliación de la noción de la llamada "educación básica", que antes estaba limitada sólo a niños y adolescentes, en función de contenidos de lectura, escritura y cálculo. Por ello, la insistencia está hoy no tanto en los contenidos, como en el desarrollo de competencias que nos permitan "aprender a aprender", que es la base para poder seguir aprendiendo a lo largo de toda nuestra vida.

Por tal razón, el paradigma de la complejidad nos permite ir más allá de un simple cambio o enriquecimiento de los contenidos o a las metodologías educativas. La idea de la complejidad exige ahondar en el pensamiento, en la construcción epistemológica del saber, en una educación para la comprensión, en repensar el sentido del conocimiento y el significado de la educación.

La formación humana no puede ser vista desde un solo aspecto ni simplemente como la suma desestructurada de esfuerzos diversos. La tarea educativa ha estado confiada tradicionalmente a los educadores, pero es una responsabilidad de la sociedad en su conjunto. La complejidad de lo social exige que la educación sea asumida desde una perspectiva ínter y transdiciplinaria, buscando la sinergia entre los aportes de diversos campos de estudio y de acción (pedagogía, sociología, psicología, antropología, etc.).

Ahora surgen nuevas tendencias del quehacer educativo, mediante investigaciones como las nuevas concepciones de las múltiples inteligencias, el sentido humano, el valor de la autoestima y las expectativas sociales, el entramado organizacional y cultural de los centros educativos, el valor de las emociones, intuiciones y afectos, etc. Falta, sin embargo, que la tarea educativa en sí se beneficie de la perspectiva ínter o transdiciplinaria, que se ve cada vez con más frecuencia y recursividad en el ámbito de la investigación. Esto exige, como punto clave, examinar lo que es la formación inicial y continua de los docentes.

\section{Una educación tolerante en un mundo divergente}

El Informe Delors de la Unesco (1997) define la educación en función de cuatro pilares: aprender a ser, aprender a hacer, aprender a conocer y aprender a convivir

"Aprender a convivir significa desarrollo de la tolerancia, y aún más: la apertura y aceptación de lo distinto. Convivir no puede significar una mirada reduccionista que incluye al otro en las categorías de lo extraño, lo folklórico o lo ausente. La apertura a la diversidad va mucho más allá que eso, pues supone una dimensión ética, y una convicción de que el otro es irreductible y que sin el otro yo no puedo ser yo mismo. El reconocimiento de la diversidad exige también repensar la propia identidad. Necesitamos reconocer nuestra identidad y pertenencia local, pero abiertos a las exigencias de la ciudadanía global, evitando falsas dicotomías. Tal como señala Ceruti, una de las tareas urgentes de la educación es "ayudar al individuo a percibirse como una identidad múltiple, ayudándolo al mismo tiempo a percibir a los otros individuos como identidades también múltiples. Sólo este juego de reconocimiento recíproco, en sí mismo y en los otros, puede hacer emerger nuevas ideas de ciudadanía" (Unesco, 1997) 
En el contexto de cambios y transformaciones que vivimos, debemos aprender a convivir con la incertidumbre. En los centros educativos no se deberían enseñar las cosas como definidas, leyes inmutables, versiones acabadas o doctrinas únicas. No sólo se trata de mostrar la diversidad de opiniones o puntos de vista que existen en diversas áreas del saber humano, sino también se trata de asumir con sentido crítico temas controversiales que han aparecido en las ciencias físicas (microfísica, termodinámica, cosmología), en la biología y en las ciencias históricas. Se trata de escrutar los principios que permiten afrontar lo inesperado, lo incierto, y de aprender a moverse en el terreno de arenas movedizas que supone la información fragmentada y contradictoria, que es la única con la que muchas veces contamos en la vida cotidiana. Como dice Morin, "es necesario aprender a navegar en un océano de incertidumbres a través de archipiélagos de certeza" (Morin, 1999: 58).

El objetivo del nuevo paradigma educativo consiste en formar una comunidad educativa nueva afincada en la investigación, la comunicación valorativa y la creatividad (www.edgarmorin.org). A esto ha contribuido el cuestionamiento serio del paradigma de la "transmisión de conocimientos". El hecho de que el maestro deje de ser la única fuente de conocimiento en el aula, reconozca los saberes de los alumnos, permita la irrupción de nuevas fuentes de conocimiento y la construcción de saberes, es de por sí un giro copernicano en el contexto educativo.

Los maestros son los primeros que deben aprender a asumir la incertidumbre como una realidad cotidiana. Esta actitud ayuda a ser auténticos compañeros de camino de los estudiantes que transitan por la vida en la búsqueda de respuestas esenciales. Como señala Gutiérrez, "el profesor no enseña, sino que ayuda a aprender, y el aprendizaje es autopoiesis" (Gutiérrez, 2001)4.

$4 \quad$ La autopoiesis es la capacidad de un sistema para autoorganizarse, lo cual implica que el conocimiento del estudiante es sujeto y objeto de su propio proceso de indagación.
Entonces, la educación debe suponer una extensión del horizonte, una apertura permanente hacia lo nuevo, lo cual implica estar dispuestos para lo inesperado, evitando la trivialidad y el determinismo empobrecedor. Como dice Morin, "la complejidad no es una receta para conocer lo inesperado [...] El pensamiento complejo no resuelve, en sí mismo, los problemas pero constituye una ayuda para la estrategia que puede resolverlos" (2001)

\section{La educación, una carrera hacia el futuro}

Estas son algunas de las implicaciones del pensamiento complejo sobre la educación, pero no agotan el tema. Morin señala que "lo complejo no puede resumir el término complejidad, retrotraerse a una ley de complejidad, reducirse a la idea de complejidad" (Morin, 2001). La complejidad es el paradigma en que nos movemos, por tanto, supone afrontar una tensión entre la "aspiración a un saber no parcelado, no dividido, no reduccionista, y el reconocimiento de lo inacabado e incompleto de todo conocimiento" (Morin, 2001).

La educación es y seguirá siendo un campo particular donde se expresen estas tensiones, y en el que las propuestas particulares, los intereses privados y colectivos seguirán expresándose de formas diversas. Quizás no se han ponderado suficientemente lo que se plantea en la visión para la educación para el futuro cercano: la preocupación por la educación nos lleva a indagar sobre las maneras como aprendemos los conocimientos y asimilamos valores, como inventamos comportamientos diferentes y construimos nuevos proyectos de vida colectivos. En la medida que la indagación se convierta en actitud permanente, podremos estar a las puertas de una reforma que sea significativa. Como afirma Raúl Motta, "no es posible una verdadera reforma de la educación sin una reforma del pensamiento, cuyos primeros pasos implican una ecología de la inteligencia colectiva y una ecología de las ideas" (1999). Entonces, se necesita el desarrollo de una nueva sensibilidad, una nueva mentalidad y nuevas actitudes, que 
permitan ver lejos y, al mismo tiempo, mirar en lo profundo de las vivencias.

Lograr una educación de calidad que sea a la vez equitativa es uno de los retos más significativos de nuestro tiempo. Así lo ha reconocido el Foro Mundial que se realizó en Dakar en 2000. Sin embargo, una de las debilidades fundamentales de nuestros sistemas educativos es que muchas veces no hay condiciones para que los actores de la educación puedan ir resolviendo, en la práctica cotidiana, los problemas que se presentan. Algunos creen que esto será posible sólo si se impulsa la creación de comunidades de aprendizaje en las escuelas, los colegios y las universidades. En otras palabras, es urgente que tanto directivos como docentes, con el apoyo de los padres de familia y la comunidad en general, compartan una misma visión de su trabajo y tengan altas expectativas sobre lo que esperan de los niños, adolescentes y jóvenes promesas.

El desarrollo de comunidades de aprendizaje supone una nueva comprensión de la profesión docente, no sólo de parte de las autoridades, sino también de parte de los docentes. Como señala Rosa M. Torres (2000),

restituir a la institución escolar su función primordial, que es la enseñanza y el aprendizaje, la democratización del conocimiento, la integración y la transformación social, la identificación y el desarrollo del potencial de los alumnos, el aprender a aprender y a disfrutar del aprendizaje. Éste es el contexto institucional para el ejercicio y desarrollo de la docencia como una actividad profesional.

Nuestra apuesta es que en la medida que los docentes se integran como comunidad investigadora, puedan ir transformando efectivamente la educación y mejorando las condiciones del aprendizaje. En tal sentido, es relevante preguntarnos qué significa investigar y cómo puede ser un proceso significativo para el maestro en medio de las exigencias cotidianas de su labor docente. La investigación no es una tarea fácil en medio de la rutina y las múltiples exigencias que vive el maestro: las duras condiciones laborales, la falta de incentivos y medios apropiados convierten la investigación en todo un reto.

\section{Comunidades investigativas en el diseño del cambio}

El problema de la investigación se ha enfocado desde una perspectiva instrumental, productiva y pragmática. Enseñar a investigar sigue siendo enseñar técnicas de investigación. En realidad, cada vez hay mayor conciencia de que se requiere una aproximación más integral que aborde el tema de la cultura académica; así, la perspectiva del pensamiento complejo puede ayudar a entender mejor las actitudes que se requieren para hacer realidad las comunidades de aprendizaje y la investigación como tarea vital, holística de la realidad.

No hay investigación si no hay incertidumbre, perplejidad, confusión e, incluso, vivencia del caos. Tal vez hemos creído por mucho tiempo que definir el problema inicial de una investigación era cuestión de un poco de sentido común, algunos criterios y algo de decisión, pero en realidad no es tan fácil. Definir el problema es dejar que se instale la perplejidad, motor de la indagación crítica y el saber lógico. Es algo así como iniciar un viaje, sin tener la ruta predefinida, en el que hallamos una aventura con sorpresas, itinerarios y encuentros inesperados. Si bien la perplejidad es un asunto personal, lo cierto es que la investigación es un camino construido desde esfuerzos conjuntos: en realidad llevo las palabras aprendidas, los conceptos reconstruidos y soy heredero de una tradición. Mi pensamiento está atravesado por muchos otros. Por eso, hago el esfuerzo de reconocer desde qué campo intelectual me pregunto, me cuestiono. Por eso, cuando investigo reconozco las "huellas" de los pensamientos de otros en el mío. 
Uno de los puntos que son puestos en cuestión desde la perspectiva del pensamiento complejo es el de la objetividad. La investigación educativa que emprende el docente se da desde un distanciamiento y, a la vez, un acercamiento de los problemas. En un texto clásico, Schutz muestra que, al aproximarnos a una realidad distinta, se revelan las pautas culturales con las que tendemos a juzgar $y$ entender el mundo, y frente a ese extrañamiento somos capaces de entender lo que otros dan por hecho (Schutz, 1974).

Para investigar hay que renunciar, de antemano, a la ilusión del observador imparcial, "objetivo". Berman muestra que la realidad comienza a desempeñar un papel sólo después de que el conocimiento ha sido obtenido visceralmente (Berman, 2001). Por otra parte, en términos de Bourdieu, caemos en cuenta de que toda pregunta se hace desde un campo de poder y un campo intelectual (Bourdieu, 1983); es decir, en realidad no hay pregunta neutral ni inocente. Siempre leemos la realidad desde ciertas premisas, intereses y opciones.

Respecto de la investigación docente, es en medio de lo cotidiano desde donde los maestros deben aprender a complejizar, a no quedarse con respuestas simplistas para explicar lo que sucede. Esta es una actitud crítica difícil de lograr. El maestro debe volver sobre su experiencia diaria y tener una nueva mirada sobre ella, afrontar y reflexionar los problemas. Tal ejercicio no es un asunto individual ni aislado.

El ejercicio investigativo está inserto en la pasión y el deseo. Los maestros deben desarrollar una suerte de pensamiento empático con los aspectos de su realidad cotidiana, poner todo el interés en las vivencias y los sentidos que adquieren. No se puede educar al margen de una actitud ética por el mundo que se quiere construir. Al respecto, Humberto Maturana señala:

\footnotetext{
Yo espero que mis alumnos sean capaces de hacer cualquier cosa siendo responsables de lo que hacen, y esto exige que sean capaces de reflexionar sobre su quehacer. Pero la reflexión es un acto que exige 'soltar' lo que se tiene para ponerlo en el espacio de las emociones y mirarlo (Maturana, 1972: 238).
}

Los desafíos impuestos por el siglo XXI traen como exigencia un cambio profundo en la manera en la que se entiende la investigación y se produce el conocimiento. Esto exige revaluar el espíritu de insularidad y especialización que predominó en la ciencia tradicional, para dar paso a una concepción más abierta, dinámica e integradora del conocimiento. Desde esta perspectiva, la nueva tarea de la educación consiste en ayudar a comprender la realidad como una totalidad compleja, haciendo visibles sus relaciones e interacciones. 


\section{Bibliografía}

Berman, M. (2001). El reencantamiento del mundo. Santiago de Chile: Cuatro Vientos.

Bordieu, P. (1983). Campo de Poder y Campo Intelectual. Buenos Aires: Folios.

Ceruti, M. (1998). Educación, desafío de la complejidad y desafío de la globalización. Universidad de Milán-Bicocca.

Comisión Europea (2000). Memorándum sobre el aprendizaje permanente. Bruselas.

Delors, J. et al. (1997). La Educación encierra un tesoro. Lima: Santillana.

Gutiérrez, G. (2001). Por los caminos del conocimiento. Notas sobre los nuevos paradigmas en gnoseología, en: Umbral 2000, №. 6, 2001, Santiago de Chile, disponible en Internet: http://dialnet.unirioja.es/servlet/articulo?codig $\mathrm{o}=124931$ \&orden $=48004$ \&info $=$ link

Latorre, H. y Suárez, P. (2000). La evaluación escolar como mediación. Bogotá: Orión.

Maturana, H. (1972). El sentido de lo humano. Santiago: Hachette.

Morin, E. (2001). Introducción al pensamiento complejo. En: Curso Pensamiento Complejo. Selección de textos realizado por Gonzalo Gutiérrez. Santiago de Chile: EDUC.
Morin, E. Los siete saberes necesarios para la educación del futuro. París: UNESCO.

Motta, R. (1999). Complejidad, Educación y Transdisciplinariedad. Buenos Aires.

Páramo, G. (1989). Lógica de los mitos: Lógica Paraconsistente. En: Ideas y Valores, № 79, Abril de 1989.

Pozo, I. (1999). Aprendices y maestros: La nueva cultura del aprendizaje. Madrid: Alianza.

Ramón, M. A. (1999). La universidad en la sociedad del conocimiento: Conferencia Internacional de Educación a Distancia, la solución educativa para el siglo XXI. Bogotá: Instituto Colombiano para el Fomento de la Educación Superior.

Schutz, A. (1974). El Forastero: Ensayo de Psicología Social. En: Schutz, A. Estudios sobre teoría social. Buenos Aires: Amorrortu.

Torres, R. M. (2000). De agentes de la reforma a sujetos del cambio. En: Perspectivas, vol. XXX, n² 2, junio 2000.

www.edgarmorin.com 